\title{
Measurement of the neutron electric form factor in quasielastic electron scattering
}

\author{
Björn Sören Schlimme* on behalf of the A1 collaboration \\ Institut für Kernphysik, Johannes Gutenberg-Universität Mainz, \\ Johann-Joachim-Becher-Weg 45, 55128 Mainz, Germany \\ E-mail: schlimme@kph.uni-mainz.de
}

The electric form factor of the neutron, $G_{\mathrm{E}}^{\mathrm{n}}$, has been measured at a momentum transfer $Q^{2}=$ $1.58(\mathrm{GeV} / c)^{2}$ at the Mainz Microtron. Longitudinally polarized electrons were scattered on a polarized ${ }^{3} \mathrm{He}$ target, which was used as an effective polarized neutron target. The scattered electrons were detected in a magnetic spectrometer in coincidence with neutrons detected in a neutron detector.

$G_{\mathrm{E}}^{\mathrm{n}}$ was determined from double polarization observables in the studied quasielastic scattering process ${ }^{3} \mathrm{He}\left(\overrightarrow{\mathrm{e}}, \mathrm{e}^{\prime} \mathrm{n}\right) \mathrm{pp}$. Cross section asymmetries with respect to the electron helicity are sensitive to the electric to magnetic form factor ratio $G_{\mathrm{E}}^{\mathrm{n}} / G_{\mathrm{M}}^{\mathrm{n}}$ if the target polarization is aligned perpendicular to the momentum transfer. Asymmetries measured with an alignment parallel are used for a reduction of systematics. With the magnetic form factor $G_{\mathrm{M}}^{\mathrm{n}}$ being comparatively well known, a determination of $G_{\mathrm{E}}^{\mathrm{n}}$ can be provided.

50th International Winter Meeting on Nuclear Physics

23-27 January 2012

Bormio, Italy

\footnotetext{
*Speaker.
} 


\section{Electromagnetic nucleon form factors}

Elastic electron scattering has a great capability to probe the internal electromagnetic structure of the nucleon. In the Born approximation the electromagnetic interaction between the electron and the target particle can be described by the exchange of one virtual photon with invariant mass squared $q^{2}=\omega^{2}-\vec{q}^{2}$. Here $\vec{q}$ is the three-momentum transfer and $\omega$ the energy transfer in the scattering process. The cross section for elastic electron scattering on a point like spin-1/2 particle can be described by the Mott cross section. In contrast two form factors (FF) are additionally required to parametrize the scattering process on a nucleon due to its internal structure. These FF belong to the most basic observables of the nucleon. Precise measurements can be used to test nonperturbative QCD and to constrain phenomenological models of the nucleon structure.

For a specific parametrization, the so-called electric and magnetic Sachs FF $G_{\mathrm{E}}\left(Q^{2}\right)$ and $G_{\mathrm{M}}\left(Q^{2}\right)$ characterize the electric and magnetic distributions of the nucleon. They are functions of $Q^{2} \equiv-q^{2}$. For low momentum transfer, $G_{\mathrm{E}}$ and $G_{\mathrm{M}}$ can be interpreted as the fourier transforms of the spatial charge and the current distributions in the nucleon.

\section{Measurement technique}

The experimental determination of the neutron FF suffers from the absence of a free neutron target that could be used in electron scattering experiments. Nevertheless, scattering experiments on light nuclei like ${ }^{2} \mathrm{H}$ and ${ }^{3} \mathrm{He}$ can be used to deduce information on the neutron FF. Both elastic scattering on the nucleus and quasielastic scattering on the bound nucleons can be exploited. Naturally the presence of the protons contaminates the interesting observables and makes it desirable to distinguish between contributions from the two nucleon species. Furthermore nuclear binding effects complicate the situation. Different experimental approaches have been developed, see [1] for instance for a review on nucleon form factors including the description of different measurement methods.

The magnetic FF of the neutron, $G_{\mathrm{M}}^{\mathrm{n}}$, has been determined with a precision in the order of a few percent up to moderate high $Q^{2}$ values (see [2] and references there). The fact that the electric neutron FF $G_{\mathrm{E}}^{\mathrm{n}}$ is much smaller than $G_{\mathrm{M}}^{\mathrm{n}}$ in the $Q^{2}$-range explored up to today greatly limits the precision with which $G_{\mathrm{E}}^{\mathrm{n}}$ can be measured in unpolarized quasielastic scattering. This is because the cross section is strongly dominated by the contribution of the magnetic FF in that case. Alternative techniques using polarization degrees of freedom have emerged for that reason. Observables in these measurements can particularly be sensitive to the FF ratio $G_{\mathrm{E}}^{\mathrm{n}} / G_{\mathrm{M}}^{\mathrm{n}}$. Using $G_{\mathrm{M}}^{\mathrm{n}}$ from other measurements or parametrizations, one can obtain $G_{\mathrm{E}}^{\mathrm{n}}$.

The method that was used in the experiment at hand is based on the analysis of cross section asymmetries of the fundamental process $\vec{n}\left(\vec{e}, e^{\prime} n\right)$, where longitudinally polarized electrons scatter on polarized neutrons. Again, since there is no free neutron target, a polarized ${ }^{3} \mathrm{He}$ target was used as substitute. Thanks to its special spin structure, polarized ${ }^{3} \mathrm{He}$ can serve as an effective polarized neutron target, see figure 1. Comparing count rates for different helicities of the incoming electron, one can observe the beam target asymmetry $A=\frac{N^{+}-N^{-}}{N^{+}+N^{-}}$with the luminosity corrected count rates $N^{+}$(electron spin orientated parallel to the direction of the electron beam) and $N^{-}$(antiparallel). 


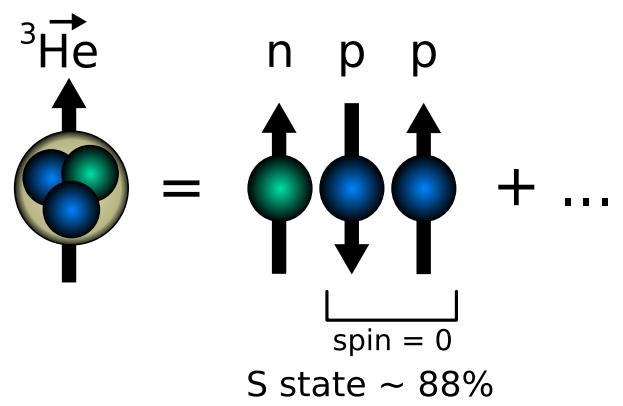

Figure 1: The ground state of ${ }^{3} \mathrm{He}$ is dominated by a symmetric $\mathrm{S}$ state. The spins of the two protons saturate and the spin of the ${ }^{3} \mathrm{He}$ is carried by the spin of the neutron [3]. The mean polarization of the neutrons and the protons relative to the polarization of ${ }^{3} \mathrm{He}$ is $0.86 \pm 0.02$ and $-0.028 \pm 0.004$, respectively [4].

In the one photon exchange approximation, the asymmetry is given for a free neutron at rest through [5]

$$
A=\frac{a G_{\mathrm{E}}^{\mathrm{n}} G_{\mathrm{M}}^{\mathrm{n}} \sin \left(\theta^{*}\right) \cos \left(\phi^{*}\right)+b G_{\mathrm{M}}^{\mathrm{n}} 2 \cos \left(\theta^{*}\right)}{c G_{\mathrm{E}}^{\mathrm{n} 2}+d G_{\mathrm{M}}^{\mathrm{n}} 2} \cdot P_{\mathrm{e}} P_{\mathrm{n}} .
$$

$a, b, c, d$ are kinematic factors (of similar magnitude for the kinematics of this experiment), $P_{\mathrm{e}}$ and $P_{\mathrm{n}}$ are the electron and the neutron polarizations, respectively. $\theta^{*}$ is the angle between the neutron polarization vector and $\vec{q}$ (see fig. 2), $\phi^{*}$ the angle between the electron scattering plane and the plane spanned by the neutron polarization vector and $\vec{q}$.

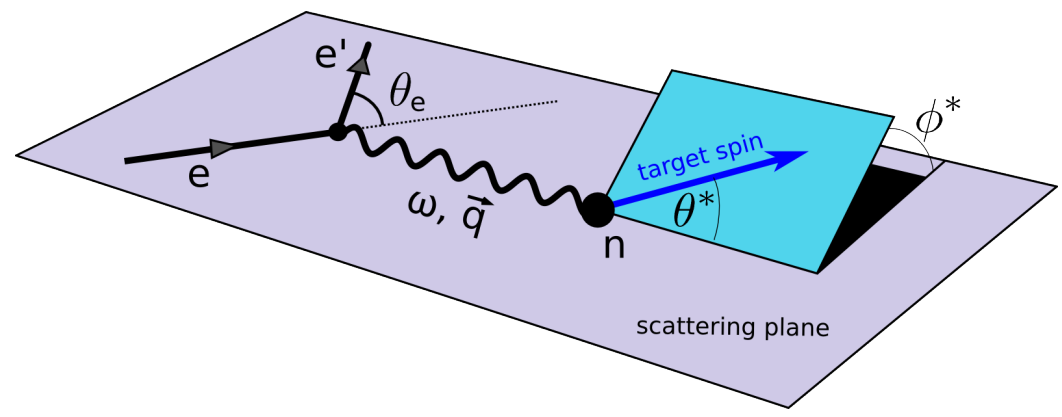

Figure 2: Definition of the angles $\theta^{*}$ and $\phi^{*}$ for the specification of the polarization orientation relative to the momentum transfer $\vec{q}$ and the scattering plane.

As is evident from eq. (2.1), measuring the asymmetry (labeled as $A_{\perp}$ ) for a target polarization orientated in the scattering plane and perpendicular to the momentum transfer (for instance $\theta^{*}=$ $90^{\circ}$ and $\phi^{*}=0^{\circ}$ ) one is sensitive to $\frac{G_{\mathrm{E}}^{\mathrm{n}}}{G_{\mathrm{M}}^{\mathrm{n}}}$. A supplemental measurement of the asymmetry $A_{\|}$for a target polarization aligned with the momentum transfer $\left(\theta^{*}=0^{\circ}\right)$ allows for the calculation of the asymmetry ratio

$$
\frac{A_{\perp}}{A_{\|}} \propto \frac{a}{b} \cdot \frac{\left[P_{\mathrm{e}} P_{\mathrm{n}}\right]_{\perp}}{\left[P_{\mathrm{e}} P_{\mathrm{n}}\right]_{\|}} \cdot \frac{G_{\mathrm{E}}^{\mathrm{n}}}{G_{\mathrm{M}}^{\mathrm{n}}}
$$

and therefore for a determination of $\frac{G_{\mathrm{E}}^{\mathrm{n}}}{G_{\mathrm{M}}^{\mathrm{n}}}$ with several benefits: 


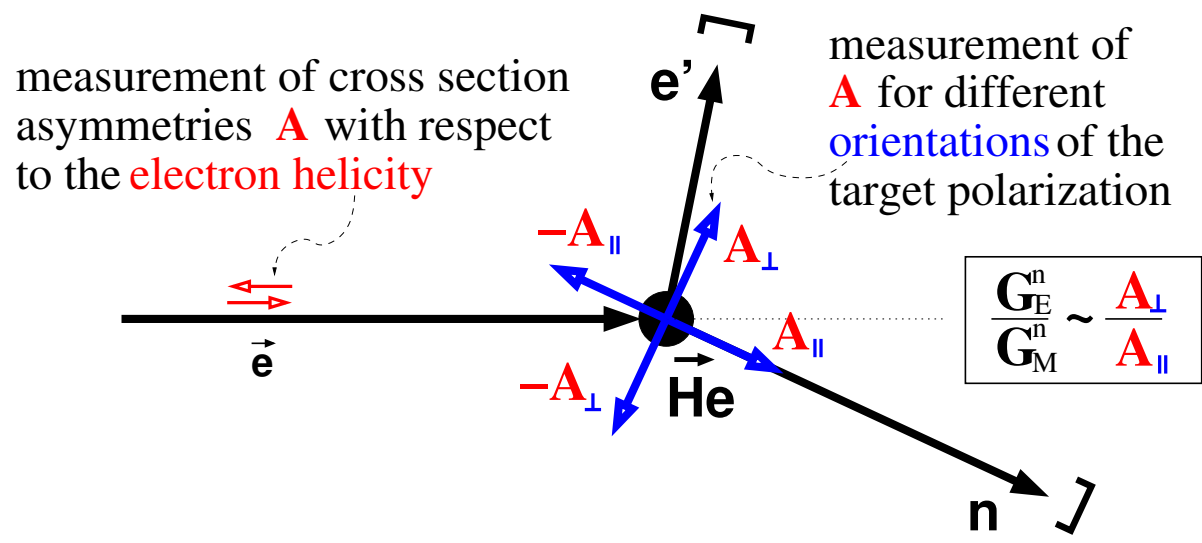

Figure 3: Measurement of beam target asymmetries for different target polarization orientations. A longitudinally polarized electron beam impinged on a polarized ${ }^{3} \mathrm{He}$ target which served as a polarized neutron target. For a separation between contributions from quasielastic scattering on the neutron and on the proton, the scattered electrons were detected in coincidence with the recoil nucleons: By means of veto layers, the neutron detector had the capability to distinguish between recoil neutrons and protons. Beam helicity asymmetries of the reaction ${ }^{3} \overrightarrow{H e}\left(\vec{e}, e^{\prime} n\right)$ pp are particularly sensitive to $\frac{G_{\mathrm{E}}^{\mathrm{n}}}{G_{\mathrm{M}}^{\mathrm{n}}}$ for a perpendicular orientation of the target polarization with respect to the momentum transfer. The measured asymmetries for an (anti)parallel alignment can be used to reduce systematics.

- Only relative polarizations have to be determined; absolute calibration factors and the relative neutron to ${ }^{3} \mathrm{He}$ polarization drop out in the fraction of the asymmetries at perpendicular $(\perp)$ and parallel $(\|)$ polarization orientations.

- Depending on the experimental conditions several dilution factors have to be taken into account for the single asymmetries. In the asymmetry ratio, the contributions from unpolarized background cancels within statistical fluctuations.

- The determination of $\frac{G_{\mathrm{E}}^{\mathrm{n}}}{G_{\mathrm{M}}^{\mathrm{n}}}$ is based on the analysis of asymmetries from quasielastic electron neutron scattering. To select scattering events on the neutron, the recoiling nucleons were detected in coincidence with the scattered electron (see below). Veto detectors were used to distinguish between the scattering on neutrons and protons. Though the recoiling protons can both be converted to neutrons in the experimental shielding or they can be misidentified as neutrons. Due to the small polarization fraction of the protons, the residual net effect of the proton contribution to the asymmetry ratio is comparatively small.

For reasons of redundance, asymmetries were also measured for $\left(\theta^{*}=180^{\circ}, \phi^{*}=0^{\circ}\right)$ and $\left(\theta^{*}=\right.$ $270^{\circ}, \phi^{*}=0^{\circ}$ ), see fig. 3 .

\section{Experimental setup}

The experiment was carried out at the electron accelerator facility MAMI [6]. It consists of three stages of race track microtrons. A fourth stage MAMI-C [7], comprised of a harmonic double-sided microtron, permits beam energies of up to $1.6 \mathrm{GeV}$. It provided $1.508 \mathrm{GeV}$ longitudinally polarized electrons with a beam current around $10 \mu \mathrm{A}$. The electron helicity was flipped 

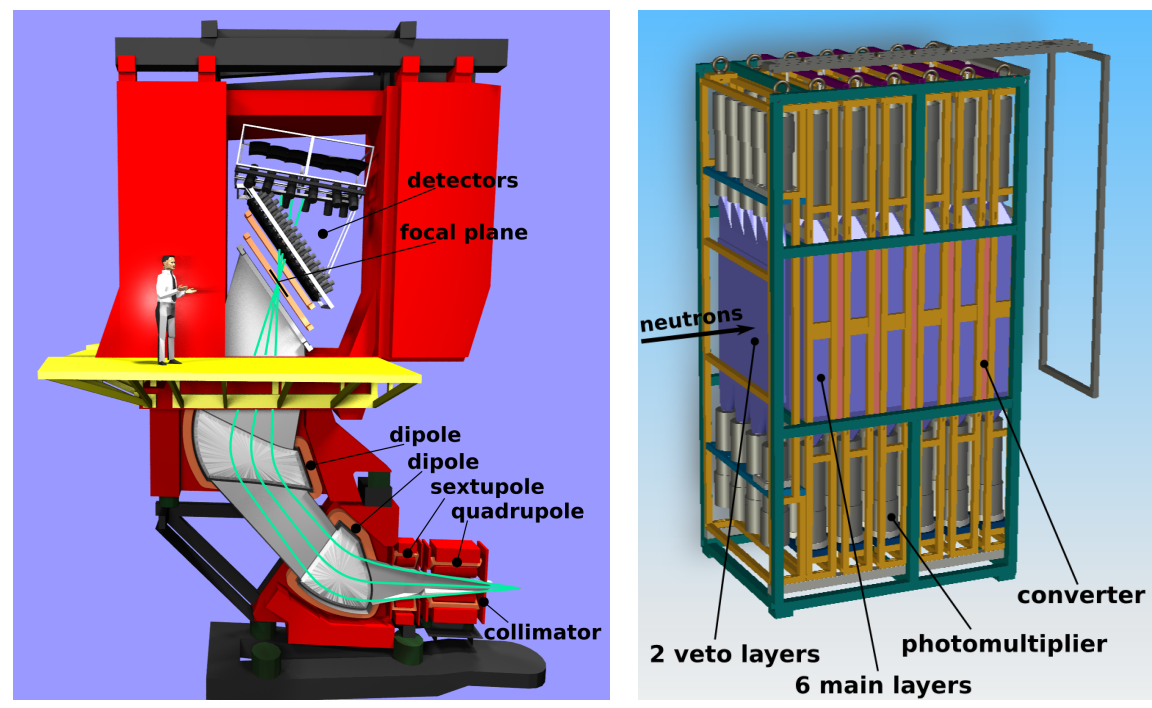

Figure 4: Left: Spektrometer A has been used for the detection of the scattered electrons. It is a high resolution magnetic spectrometer. The detector system comprises of vertical drift chambers, scintillator paddles and a Čerenkov threshold detector for the distinction between electrons and pions. Right: An array of scintillator bars was used as neutron detector. Thin veto layers in front of the detector were used for charged particle discrimination. Cupper plates (converter) were inserted between the layers for an enhancement of the neutron detection efficiency.

quasirandomly with a frequency of $1 \mathrm{~Hz}$. The beam polarization was measured twice a day using a møller polarimeter. Only minor fluctuations were found around a mean polarization of $76.3 \%$.

The experiment was set up in the spectrometer hall of the A1 collaboration [8]. The scattered electrons were detected with Spektrometer A, a high resolution magnetic spectrometer, see fig. 4 left. Two dipole magnets provide the determination of the particle momentum, a quadrupole and a sextupole magnet are used for focussing and aberration corrections. The particle track within the spectrometer can be reconstructed via two packages of vertical drift chambers, the particle momentum at the target can be deduced by the use of transfer matrices. Two layers of scintillator bars serve for triggering and timing issues. Finally a Čerenkov detector was used for a clean electron identification.

For the detection of the recoiling nucleons a plastic scintillator array was used, see fig. 4 right. It consisted of six layers with five bars $\left(50 \times 10 \times 10 \mathrm{~cm}^{3}\right)$. Each bar was equipped with two photomultiplier tubes. For a rejection of charged particles, two $1 \mathrm{~cm}$ thin veto layers were installed additionally in front of the detector. The neutron detector was heavily shielded with $5 \mathrm{~cm}$ boron-treated polyethylene (outer shielding) and $10 \mathrm{~cm}$ lead (inner shielding) except for the entrance window pointing to the target. The latter was only shielded with $1 \mathrm{~cm}$ lead to keep the proton to neutron conversion probability small for protons coming from the target.

As target a highly polarized 5 bar ${ }^{3} \mathrm{He}$ gas target was used. It was polarized outside the experimental hall using the $M E O P$ technique with an initial degree of polarization of up to $72 \%$ [9]. The gas was stored in cesium coated quartz glass cells and was transported to the target area. The cells were put in a magnetically shielded target box which provided a magnetic guiding field 
$\left(\approx 4 \cdot 10^{-4} \mathrm{~T}\right)$. By altering the direction of this field, the direction of the target polarization could be freely oriented. The relaxation time was about 30 to 40 hours under beam conditions. Therefore the target cell was changed twice a day resulting in a mean target polarization of $55.6 \%$.

The central kinematics of this experiment is summarized in table 1.

\begin{tabular}{|c|c|c|c|c|c|c|}
\hline $\begin{array}{c}Q^{2} \\
{\left[\mathrm{GeV}^{2} / c^{2}\right]}\end{array}$ & $\begin{array}{c}\omega \\
{[\mathrm{GeV}]}\end{array}$ & $\begin{array}{c}|\vec{q}| \\
{[\mathrm{GeV} / c]}\end{array}$ & $\begin{array}{c}k_{0} \\
{[\mathrm{GeV}]}\end{array}$ & $\begin{array}{c}k_{0}^{\prime} \\
{[\mathrm{GeV}]}\end{array}$ & $\begin{array}{c}\theta_{\mathrm{e}} \\
{\left[{ }^{\circ}\right]}\end{array}$ & $\begin{array}{c}\theta_{\mathrm{n}} \\
{\left[{ }^{\circ}\right]}\end{array}$ \\
\hline 1.58 & 0.855 & 1.52 & 1.508 & 0.653 & 78.6 & 24.9 \\
\hline
\end{tabular}

Table 1: Central kinematics of the experiment. It is $Q^{2}=-q_{\mu} q^{\mu}$ the negative of the squared four-momentum transfer, $\omega$ the energy transfer, $\vec{q}$ the three-momentum transfer, $k_{0}$ the beam energy, $k_{0}^{\prime}$ the energy of the scattered electron, $\theta_{\mathrm{e}}$ the electron scattering angle and $\theta_{\mathrm{n}}$ the angle of the knocked out neutron.

\section{Analysis}

For each recorded event the raw data of the individual detectors are considered to reconstruct kinematic quantities like the angles of the detected particles, the momentum of the scattered electron and the reaction vertex.

After electron identification the coincidence time spectrum between the electron and the neutron arm is very clean. Electron identification is mainly accomplished through the evaluation of the Čerenkov detector signal. A coincidence time resolution of $2.1 \mathrm{~ns}$ (FWHM) is achieved, the fraction of accidental coincidences is estimated to be $0.7 \%$.

To suppress inelastic events, kinematical cuts are applied as quasielastic event selection. A distinction between quasielastic scattering events on the proton and on the neutron is not feasible using information of the reconstructed kinematics since the mass of the nucleons is alike. The veto counters of the neutron detector are used therefore for a rejection of charged particles. The first layer of thick neutron detector bars is also used for charged particle rejection because parts of the veto detectors were found not to be fully efficient due to a high background level. The number of protons being misidentified as neutrons is estimated by the analysis of data taken on a hydrogen target. The relative fraction is found to be $(12.8 \pm 1.7) \%$. Since the polarization of the protons relative to the target polarization is comparatively small, the main effect of the proton background is a dilution of the single asymmetries in the order of $10 \%$. The asymmetry ratio, which is used to extract $\frac{G_{\mathrm{E}}^{\mathrm{n}}}{G_{\mathrm{M}}^{\mathrm{n}}}$, is effected much less. Using events identified as quasielastic scattering on the proton, the asymmetries of the proton background can be assessed. The correction for the proton contribution is found to be $(1.6 \pm 1.2) \%$.

Once the data selection has been performed, the analysis is based on the study of the measured asymmetries of the selected ${ }^{3} \overrightarrow{\mathrm{He}}\left(\overrightarrow{\mathrm{e}}, \mathrm{e}^{\prime} \mathrm{n}\right)$ pp events. The raw experimental asymmetries are shown in fig. 5 for the four target spin orientations.

For a better handle on variations of kinematic variables, present polarization degrees of the electron beam and the target and so forth - which effect the asymmetry (2.1) - an event-by-event analysis is performed based on a maximum likelihood fit. The reconstructed kinematics is considered explicitely for each event as well as instant polarizations. The influence of effects like 


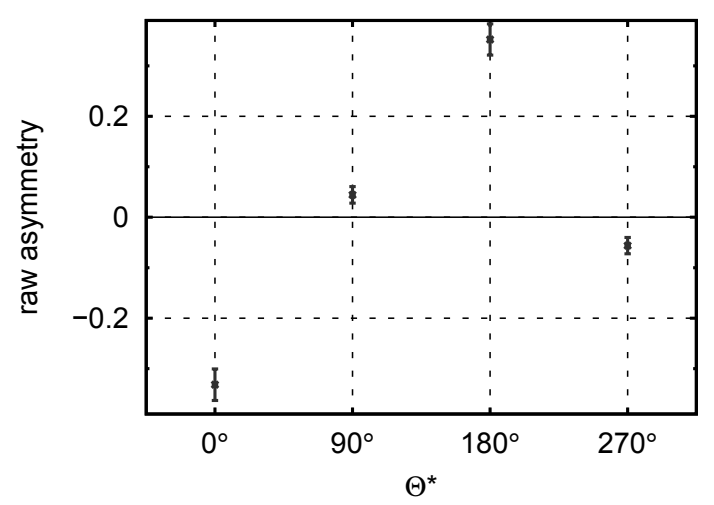

Figure 5: Raw experimental asymmetries of events from the reaction ${ }^{3} \mathrm{He}\left(\overrightarrow{\mathrm{e}}, \mathrm{e}^{\prime} \mathrm{n}\right) \mathrm{pp}$. The asymmetries for $\theta^{*}=90^{\circ}$ and $270^{\circ}$ are the ones sensitive to $\frac{G_{\mathrm{E}}^{\mathrm{n}}}{G_{\mathrm{M}}^{\mathrm{n}}}$, the asymmetries for $\theta^{*}=0^{\circ}$ and $180^{\circ}$ have been measured for a reduction of systematics.

detector resolutions and energy loss of the particles is investigated using a Monte Carlo simulation. Different contributions to the systematic uncertainty of $\frac{G_{\mathrm{E}}^{\mathrm{n}}}{G_{\mathrm{M}}^{\mathrm{n}}}$ add up to $6.4 \%$ relative, while the statistical error is $23 \%$. Theoretical corrections due to the effects of final state interactions and meson exchange currents still have to be employed; these are expected to be in the order of only a few percent. For a determination of $G_{\mathrm{E}}^{\mathrm{n}}, G_{\mathrm{M}}^{\mathrm{n}}$ data from [2] are used. The result of the analysis is shown in fig. 6.

\section{Summary and Outlook}

A measurement of the neutron electric form factor has been carried out at the Mainz Microtron in 2008. During 3 weeks of beam time, data of the reaction ${ }^{3} \mathrm{He}\left(\overrightarrow{\mathrm{e}}, \mathrm{e}^{\prime} \mathrm{n}\right) \mathrm{pp}$ has been collected. Beam helicity asymmetries for a target polarization aligned perpendicular to the momentum transfer are sensitive to the form factor ratio $\frac{G_{\mathrm{E}}^{\mathrm{n}}}{G_{\mathrm{M}}^{\mathrm{n}}}$. Asymmetries for parallel orientation are used for a reduction of systematics. Using data for $G_{\mathrm{M}}^{\mathrm{n}}, G_{\mathrm{E}}^{\mathrm{n}}$ is determined. The analysis is almost finished, nuclear corrections still have to be applied. At present a new highly segmented, high performance neutron detector is being developed within the A1 collaboration. This detector is planned to be used in a systematic neutron form factor measurement campaign in the $Q^{2}$ range from 0.2 to $1.5(\mathrm{GeV} / c)^{2}$ [10].

\section{Acknowledgments}

We gratefully acknowledge the essential support from the technical staff at the Mainz Microtron and thank the accelerator group for the excellent beam quality. Work supported by the "Deutsche Forschungsgemeinschaft (DFG)" with a Collaborative Research Centre (SFB 443). 


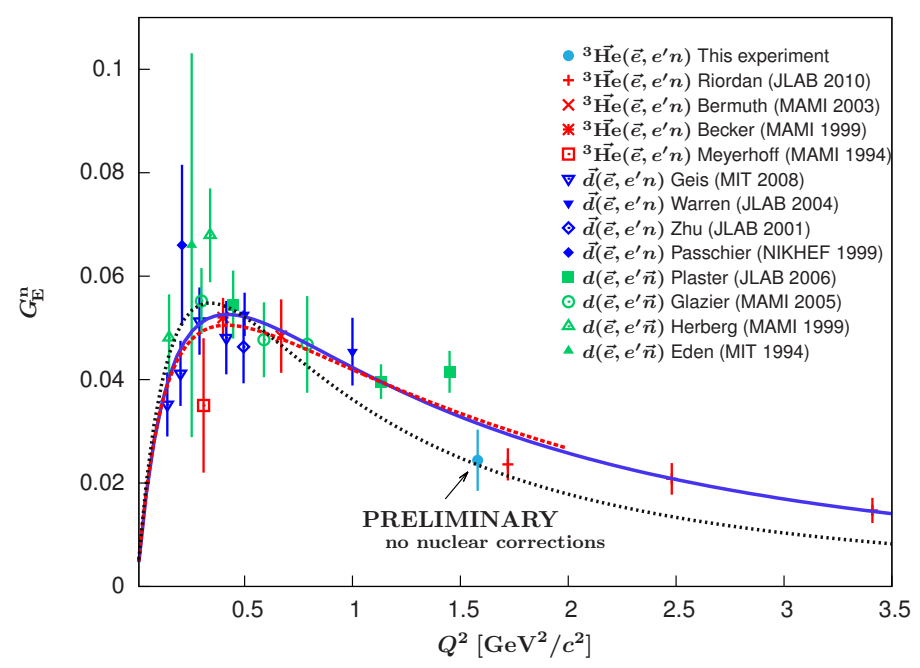

Figure 6: The result of this experiment (full circle) compared to data from other double polarization experiments [11-22]. The error bars shown are statistical and systematic uncertainties added in quadrature. In these experiments the reaction ${ }^{3} \overrightarrow{\mathrm{He}}\left(\overrightarrow{\mathrm{e}}, \mathrm{e}^{\prime} \mathrm{n}\right)$ was studied, a polarized deuteron target was employed or the neutron recoil polarization was measured instead of the usage of a polarized target. Shown is also the result of a two-dipole fit [23] (solid line), the result of a dispersion analysis [24] (dashed) and the so-called Galster parametrization [25] (dotted).

\section{References}

[1] C.F. Perdrisat, V. Punjabi and M. Vanderhaeghen, Nucleon electromagnetic form factors, Prog. Part. Nucl. Phys. 59 (2007) 694.

[2] J. Lachniet et al., Precise Measurement of the Neutron Magnetic Form Factor $G_{M}^{n}$ in the Few-GeV ${ }^{2}$ Region, Phys. Rev. Lett. 102 (2009) 192001.

[3] B. Blankleider and R.M. Woloshyn, Quasi-elastic scattering of polarized electrons on polarized ${ }^{3}$ He, Phys. Rev. C 29 (1984) 538.

[4] S. Scopetta, Neutron single spin asymmetries from semi-inclusive deep inelastic scattering off transversely polarized ${ }^{3}$ He, Phys. Rev. D 75 (2007) 054005.

[5] T.W. Donelly and A.S. Raskin, Considerations of Polarization in Inclusive Electron Scattering from Nuclei, Ann. Phys. 169 (1986) 247.

[6] H. Herminghaus et al., The Design of a Cascaded 800-MeV Normal Conducting CW Racetrack Microtron, Nucl. Instrum. Methods 138 (1976) 1.

[7] K.H. Kaiser et al., The 1.5-GeV harmonic double-sided microtron at Mainz University, Nucl. Instrum. Methods A $\mathbf{5 9 3}$ (2008) 159.

[8] K.I. Blomqvist et al., The three-spectrometer facility at the Mainz microtron MAMI, Nucl. Instrum. Methods A 403 (1998) 263.

[9] J. Krimmer et al., A highly polarized He-3 target for the electron beam at MAMI, Nucl. Instrum. Methods A 611 (2009) 18. 
[10] A. Denig, M. Vanderhaeghen et al., The Low-Energy Frontier of the Standard Model, Proposal for the establishment and funding of Collaborative Research Centre 1044 (2011).

[11] S. Riordan et al., Measurements of the Electric Form Factor of the Neutron up to $Q^{2}=3.4 \mathrm{GeV}^{2}$ Using the Reaction ${ }^{3} \mathrm{He}\left(\vec{e}, e^{\prime} n\right) p p$, Phys. Rev. Lett. 105 (2010) 262302.

[12] J. Bermuth et al., The neutron charge form factor and target analyzing powers from ${ }^{3} \overrightarrow{\mathrm{He}}\left(\overrightarrow{\mathrm{e}}, \mathrm{e}^{\prime} \mathrm{n}\right)$ scattering, Phys. Lett. B 564 (2003) 199.

[13] J. Becker et al., Determination of the neutron electric form factor from the reaction ${ }^{3} \mathrm{He}\left(e, e^{\prime} n\right)$ at medium momentum transfer, Eur. Phys. J. A 6 (1999) 329.

[14] M. Meyerhoff et al., First measurement of the electric formfactor of the neutron in the exclusive quasielastic scattering of polarized electrons from polarized ${ }^{3}$ He, Phys. Lett. B 327 (1994) 201.

[15] B. Plaster et al., Measurements of the neutron electric to magnetic form factor ratio $G_{E n} / G_{M n}$ via the ${ }^{2} \mathrm{H}\left(\vec{e}, e^{\prime} \vec{n}\right){ }^{1} \mathrm{H}$ reaction to $Q^{2}=1.45(\mathrm{GeV} / \mathrm{c})^{2}$, Phys. Rev. C 73 (2006) 025205.

[16] D.I. Glazier et al., Measurement of the electric form factor of the neutron at $Q^{2}=0.3-0.8(\mathrm{GeV} / \mathrm{c})^{2}$, Eur. Phys. J. A 24 (2005) 101.

[17] C. Herberg et al., Determination of the neutron electric form factor in the $D\left(e, e^{\prime} n\right) p$ reaction and the influence of nuclear binding, Eur. Phys. J. A 5 (1999) 131.

[18] T. Eden et al., Electric form factor of the neutron from the ${ }^{2} H\left(\vec{e}, e^{\prime} \vec{n}\right)^{1} H$ reaction at $Q^{2}=0.255(\mathrm{GeV} / \mathrm{c})^{2}$, Phys. Rev. C 50 (1994) R1749.

[19] E. Geis et al., Charge Form Factor of the Neutron at Low Momentum Transfer from the ${ }^{2} \overrightarrow{\mathrm{H}}\left(\vec{e}, e^{\prime} n\right)^{1} \mathrm{H}$ Reaction, Phys. Rev. Lett. 101 (2008) 042501.

[20] G. Warren et al., Measurement of the Electric Form Factor of the Neutron at $Q^{2}=0.5$ and 1.0 $G e V^{2} / c^{2}$, Phys. Rev. Lett. 92 (2004) 042301.

[21] H. Zhu et al., Measurement of the Electric Form Factor of the Neutron through $\vec{d}\left(\vec{e}, e^{\prime} n\right) p$ at $Q^{2}=0.5(\mathrm{GeV} / \mathrm{c})^{2}$, Phys. Rev. Lett. 87 (2001) 081801.

[22] I. Passchier et al., Charge Form Factor of the Neutron from the Reaction ${ }^{2} \overrightarrow{\mathrm{H}}\left(\vec{e}, e^{\prime} n\right) p$, Phys. Rev. Lett. 82 (1999) 4988.

[23] T.R. Gentile and C.B. Crawford, Neutron charge radius and the neutron electric form factor, Phys. Rev. C 83 (2011) 055203.

[24] M.A. Belushkin, H.W. Hammer and U.G. Meissner, Dispersion analysis of the nucleon form factors including meson continua, Phys. Rev. C 75 (2007) 035202.

[25] S. Galster et al., Elastic electron-deuteron scattering and the electric neutron form factor at four-momentum transfers $5 \mathrm{fm}^{-2}<q^{2}<14 \mathrm{fm}^{-2}$, Nucl. Phys. B 32 (1971) 221. 